\title{
Natural History of Barrett's Esophagus
}

\author{
Ernst J. Kuipers ${ }^{1} \cdot$ Manon C. Spaander ${ }^{1}$
}

Published online: 15 June 2018

(c) The Author(s) 2018

\begin{abstract}
Barrett's esophagus (BE) is a very common condition. We have obtained fairly profound knowledge of the natural history of this condition. This results from many cross-sectional and cohort studies, many describing patients undergoing long-term surveillance. Their consent to use their clinical data has improved our knowledge to the benefit of these same and other patients. The prevalence of BE increases with age both in men and in women. This increase starts at a younger age in men than in women. The incidence of high-grade dysplasia and cancer in BE depends on segment length, gender, and age. The latter two likely indicate the duration of the presence of BE in an individual patient. Other factors that influence the incidence of dysplasia and cancer are smoking behavior and use of certain medications such as PPIs, statins, and NSAIDs. Surveillance of BE and treatment of dysplasia can impact the incidence of and mortality due to esophageal adenocarcinoma. This is of major benefit to a subgroup of BE patients. The epidemiology and burden of disease ask for further efforts to develop targeted screening, surveillance, and intervention techniques in coming years.
\end{abstract}

Keywords Barrett's esophagus $\cdot$ Esophageal adenocarcinoma $\cdot$ Surveillance $\cdot$ Progression $\cdot$ Epidemiology $\cdot$ Prevention

\section{Introduction}

Almost one quarter of all human cancers arise in the gastrointestinal tract, the predominant cancer-affected tract in the human body. Remarkably, nearly all these malignancies are for long preceded by precursor lesions that can be visualized in detail with modern techniques. This provides major opportunities for screening, surveillance, and targeted intervention. As a result, most physicians managing patients with gastrointestinal disorders are used to deal with premalignant lesions, although the actual risks associated with different conditions are not always fully understood. The high cancer incidence in the gastrointestinal tract relates to a variety of factors. These include surface area, tissue turnover, and frequent exposure to internal and external factors that may induce tissue damage and chronic inflammation. This is all best illustrated at the gastroesophageal junction, arguably the segment of the gastrointestinal tract with the highest cancer incidence per segment length. Of note, this remarkably high cancer incidence

Ernst J. Kuipers

e.j.kuipers@erasmusmc.nl

1 Department of Gastroenterology and Hepatology, Erasmus MC University Medical Center, P.O. Box 2040, 3000 CA Rotterdam, The Netherlands is of recent date and seems to occur in many populations worldwide although at different moments in time. These differences relate to changes in lifestyle and risk factors for Barrett's esophagus (BE) and esophageal adenocarcinoma (EAC).

\section{Epidemiology of Barrett's Esophagus}

Exposure to gastroduodenal refluxate including acid and bile often leads to gastroesophageal reflux disease (GERD). This condition is among others characterized by chronic inflammation of the lining of the lower esophagus. GERD is very common in Western populations and increasing elsewhere such as in the far east $[2,11]$. It is the most common GI-related diagnosis in the USA [1]. A systematic review of 28 studies from different countries reported that the prevalence of GERD in adult populations ranged between 18 and 28\% in North America, 9-26\% in Europe, $9-33 \%$ in the Middle East, and 2.5-8\% in East Asia [2]. This prevalence had increased with an approximate $50 \%$ in 20 years. The same study also included two studies reporting a GERD incidence of 4.5-5.4 per 1000 person-years in the UK and USA [2]. This explains that acid-suppressive drugs, in particular proton pump inhibitors, are since long among the most commonly used drugs in the Western world. 
This increase in GERD is accompanied by a rapid increase in prevalence of Barrett's esophagus, the main target for prevention of esophageal adenocarcinoma. The exact prevalence of $\mathrm{BE}$ in different populations is difficult to assess because the condition by itself is asymptomatic, and a diagnosis is made only when an endoscopy is performed. The latter is usually done for persistent GERD symptoms, which creates a selection bias. However, there are various data that provide solid ground for the assumption that Barrett's metaplasia is a common condition in adults in populations with a high prevalence of GERD. Almost 30 years ago, a first autopsy study reported seven $(0.95 \%)$ BE cases in 733 unselected autopsies in the USA [12]. Only five cases were diagnosed at autopsy, a first indication that the majority of BE cases remain undiagnosed during their lifetime. Further information on the prevalence of BE came from endoscopy studies. In a large Dutch primary care database covering more than 500,000 subjects, we noted that patients were newly diagnosed with Barrett's esophagus at a rate of 40.5 per 1000 upper gastrointestinal endoscopies in 2002 [13]. This rate had markedly increased over time. A recent renewed analysis of the same, now expanded database in combination with similar data from the UK, showed that the rate of newly diagnosed BE cases had stabilized since the previous publication [14]. Similar changes over time are now occurring in Asia. A recent analysis of 51 endoscopy studies, mainly from Eastern Asia, including 453,147 patients noted a pooled prevalence of endoscopic Barrett's esophagus of 7.8\% (95\% CI 5.0-12.1) and of histologically confirmed Barrett's metaplasia of $1.3 \%$ $(0.7-2.2)$ [15]. These data are in line with other series. It should be noted that these were procedures for clinical indications, and thus included the major confounder of procedures being performed for GERD symptoms. In order to overcome this confounder, some studied the prevalence of $\mathrm{BE}$ in subjects undergoing screening colonoscopy. In such a study from the USA, a $6.8 \%$ prevalence was found in 961 subjects [16]. In another US study investigating 300 subjects above the age of 64 years referred for screening colonoscopy, a $16.7 \%$ prevalence of BE was observed [17]. In a similar Dutch study, we observed an approximate $7 \%$ prevalence of Barrett's esophagus among 382 colonoscopy screenees with an average age of 53 years (results unpublished).

Several population-based studies have provided further insights. Among 11 studies reporting the prevalence of upper GI pathology in general populations, three reported on the prevalence of Barrett's esophagus [18]. The first was the Swedish Kalixanda study. Among 1000 Swedish adults undergoing upper gastrointestinal endoscopy as part of a population prevalence study, an endoscopic BE segment was observed in $10.3 \%$ of the subjects. In $9.9 \%$, histology confirmed the presence of columnar epithelium, while specialized intestinal metaplasia was observed in $1.6 \%$ of them. Of those subjects, $1.1 \%$ had a short BE segment and $0.5 \%$ a long segment [19]. A similar study from Italy, the Loiano-Monghidoro study, found a 3.6\% prevalence of Barrett's esophagus in 1033 subjects, which was histologically confirmed in $1.3 \%$ [20]. The Chinese SILC study included 1030 subjects, of whom $1.8 \%$ had endoscopical signs of Barrett's esophagus [21].

More detail on the prevalence of Barrett's esophagus in different populations is provided by studies on age- and sex-related distribution of Barrett's esophagus prevalence. A study from the UK on 21,899 first endoscopies observed that the prevalence of $\mathrm{BE}$ rose with $7.4 \%$ for each additional year of age between the age of 20 and 59 year in males. It showed a similar pattern albeit with a 20-year delay in females [4]. This pattern was confirmed in a Dutch primary care population as well as in Northern Ireland [22, 23].

\section{Predisposing Factors to Barrett's Esophagus}

Gastroesophageal reflux is considered the predominant risk factor for Barrett's esophagus. The risk seems to correlate with the severity and duration of GERD. A study from the USA based on cross-sectional data from 683 Veterans Affairs patients reported that onset of GERD before the age of 30 years and the presence of weekly symptoms were together associated with a 15-fold increased risk of Barrett's esophagus [3]. Other factors include male gender, white race, cigarette smoking, and central adiposity [4, 5]. Many studies have also reported an inverse correlation between colonization with Helicobacter pylori and presence of Barrett's esophagus [3]. These factors interplay with genetic predisposition. Barrett's esophagus shows a certain degree of familial clustering. In a Dutch survey of 603 patients, definite familial clustering was found in $7 \%$ of patients [6]. This was likely an underestimate of the true prevalence of familial clustering, since a further $44 \%$ of participants reported that familial co-occurrence was either unknown or likely. Recent large population case-control studies identified more than 20 genetic variants predisposing for Barrett's esophagus in populations of European ancestry [7, 8].

\section{Risk Prediction}

Together, this knowledge led to a range of tools to predict the presence of Barrett's esophagus in specific populations. Most of these were based on demographic and clinical data alone. These models tend to have fair performance in predicting the likelihood that a subject will have Barrett's esophagus. A recent study aimed to combine these with familial history [9]. Based on close to 900 Barrett's cases, the authors developed a model using eight risk factors including age, sex, smoking, heartburn frequency, and use of acid suppressants. They combined this with family history. For 50-year-old subjects, the model predicted Barrett's 
esophagus prevalences ranging between 3 and $33 \%$ in men, and 0.5 and $10 \%$ in women [9]. A recent study went further and aimed to predict the risk of Barrett's esophagus and esophageal adenocarcinoma based on demographic and lifestyle data in combination with an individual's genetic profile [10]. The authors analyzed 3288 Barrett's cases, 2511 patients with esophageal adenocarcinoma, and 2177 controls from different cohorts. Subjects were assessed for GERD symptoms as well as age, sex, smoking habits, body mass index, and use of NSAIDs. Similar to previous models, these factors were moderately accurate to discriminate between Barrett's cases and controls with an area under the curve ranging between approximately 0.64 and 0.67 for demographics plus lifestyle factors alone and GERD history alone, and 0.79 when combining both. Each individual was further assigned a polygenic risk score based on their number of risk alleles $(0,1$, or 2$)$ for each of 23 genetic variants identified as being associated with Barrett's esophagus. For each variant, the allele number was weighted in relation to the effect estimate of the genetic variant. The sum of these weighted allele counts was then divided by 23 to yield one polygenic risk score [10]. Adding the polygenic risk score to the other factors only marginally increased prediction accuracy with an AUC of 0.799 for all factors combined. The authors rightfully concluded that the small contribution of genetic data to their prediction tool does not justify their clinical use.

\section{Progression of Barrett's Esophagus}

These data are all relevant for an adequate understanding of the natural history of Barrett's esophagus. They provide insight into the occurrence of Barrett's metaplasia in men and women, and a background to determine progression rates to dysplasia and cancer. These two then appear closely linked, as we will discuss. Similar to the development of knowledge on the epidemiology of Barrett's esophagus, the first knowledge on progression rates to dysplasia and cancer also came from endoscopy series, in particular from tertiary care centers. One of the oldest cohorts in this respect was the Rotterdam cohort. It consisted of a cohort of 166 patients diagnosed between 1973 and 1986, and followed since then. They first did not receive endoscopic surveillance; this started in 2001. The most recent analysis of this cohort included 130 patients with an average age at initial diagnosis of 62.4 years (range 14.4-92.3 years) and an average follow-up of 14.7 years (range 2.1-32.0) [24]. Thirteen patients developed high-grade dysplasia (HGD) or esophageal adenocarcinoma (EAC) during follow-up, corresponding with a rate of 1 per 151 patient-years or $0.66 \%$ per year (95\% CI 0.58-74). Many other studies came with similar data, although often with limited patient numbers and short duration of follow-up. Together, these expanding series were over time repeatedly subjected to systematic reviews and meta-analyses. These meta-analyses consistently reported progression rates to cancer varying between 2.2 and 6.3 per 1000 patient-years when focusing on all Barrett's patients [25-29]. Further reviews taught that the risk was in the low range for patients with non-dysplastic Barrett's at a rate of 3.3 (2.8-3.8) and only 1.9 per 1000 years for patients with short-segment BE [30]. A recent US and Dutch multicenter study reported no progression to high-grade dysplasia or cancer in 167 short-segment $(<1 \mathrm{~cm})$ non-dysplastic BE cases [31]. In contrast to these findings in patients with short and non-dysplastic $\mathrm{BE}$, the rate of cancer tended to be higher in patients with BE and low-grade dysplasia. A systematic review of 24 studies with 2694 patients followed for at least 2 years reported an annual progression rate to cancer of 5.4 (3.2-7.6) per 1000 years, and progression to high-grade dysplasia or cancer combined of 17.3 (9.9-24.7) per 1000 patient-years [32] (Table 1). Most of these studies relied on single pathologist's evaluation per patient. Since the diagnosis of dysplasia is investigator dependent, more stringent criteria ask for confirmation of dysplasia by a second pathologist. This often leads to down-staging of a proportion of patients. This in turn may affect disease progression rates. This was illustrated by a recent study that re-assessed low-grade dysplastic samples of $231 \mathrm{BE}$ patients [33]. Low-grade dysplasia was confirmed in 161 (70\%); the remainder was mostly downgraded to no dysplasia or indefinite for dysplasia. Two patients were upgraded to high-grade dysplasia. In patients with confirmed low-grade dysplasia, the subsequent incidence of high-grade dysplasia and EAC combined was 5.18 (3.63-7.19), and the incidence of EAC alone 2.51 (1.49-3.99) per 100 patient-years [33]. One study of $68 \mathrm{BE}$ patients with low-grade dysplasia even reported progression to EAC alone, respectively, in combination with HGD in approximately 3 and $8.5 \%$ of patients per year [34].

The highest progression rates to cancer are not surprisingly noted in patients with high-grade dysplasia. Determination of their progression rates is confounded by the fact that HGD patients form only a small minority of BE patients that nowadays routinely undergoes intervention such as endoscopic resection. This precludes determination

Table 1 Incidence per 1000 patient-years of neoplastic progression among BE patients with no dysplasia (ND) and low-grade dysplasia (LGD)

\begin{tabular}{lll}
\hline & HGD and EAC & EAC \\
\hline ND & $5.2(95 \%$ CI $4.1 ; 6.6)[50] 3.3(95 \%$ CI $2.8 ; 3.8)[30]$ \\
LGD & $17.3(95 \%$ CI $9.9 ; 24.7)$ & $5.4(95 \%$ CI $3.2 ; 7.6)[32]$ \\
& {$[32]$} &
\end{tabular}

ND and LGD $5.8(95 \%$ CI $4.7 ; 7.2)$ [50] 4.3 (95\% CI $3.4 ; 5.5)$ [50] $H G D$ high-grade dysplasia, $E A C$ esophageal adenocarcinoma 
of natural progression rates to esophageal adenocarcinoma. Nevertheless, one systematic review included four original studies of $236 \mathrm{BE}$ patients with HGD that were followed for at least 6 months without intervention. The progression rate to cancer in these patients with high-grade dysplasia was 6.58 (4.97-8.19) per 100 years [35]. This frequent diagnosis of invasive cancer in patients with high-grade dysplasia firstly reflects the co-existence of these lesions and the subtleties of histopathological diagnosis, and secondly likely also is a measure of true progression. This forms the basis for guideline recommendations to assess dysplastic samples with two pathologists, and manage high-grade dysplasia and cancer by means of expert high-resolution endoscopy and endoscopic resection if possible. This first enables further staging and may also form adequate treatment depending on histology [36-38].

\section{Factors that Influence the Natural History of Barrett's Esophagus}

\section{Barrett's Segment Length}

As mentioned, the risk of progression to HGD or EAC seems very low in patients with short-segment BE. Conversely, the risk may increase in relation to $\mathrm{BE}$ segment length. This was confirmed in a recent US study on $1175 \mathrm{BE}$ patients followed for an average 5.5 years [39]. The risk of HGD and EAC combined was $0.31 \%$ per year for those with a BE segment $<3 \mathrm{~cm}$ and increased 1.28 -fold with every extra $\mathrm{cm}$ to $2.41 \%$ for those with a BE segment $>13 \mathrm{~cm}$. These findings were supported by others [40].

\section{Proton Pump Inhibitors}

In a prospective cohort of $540 \mathrm{BE}$ patients followed for 5.2 years, treatment with a proton pump inhibitor during follow-up was associated with an hazard ratio of 0.21 (0.07-0.66) for progression to HGD or EAC [41]. A metaanalysis of this and six other studies concluded that PPI use was associated with a $71 \%$ reduction of the risk of HGD or EAC [42]. The authors further noted a trend toward a dose-response effect with a stronger protective effect with longer duration of PPI therapy.

\section{NSAIDs and Statins}

Several studies reported that both NSAIDs and statins alone and in combination also decreased the risk for progression of BE [43-45]. The individual studies reported relative risks for NSAID and statin use alone ranging for each between 0.45 and 0.65 , with one study reporting that the combination further augmented the effect leading to a hazard ratio of 0.22 [44].

\section{Tobacco Smoking}

One study from Ireland reported that smoking was associated with a hazard ratio of 2.03 (1.29-3.17) for progression to HGD or EAC [46]. Other studies reported non-significant associations between smoking and BE progression.

A recent systematic review and meta-analysis combined these studies and confirmed that the risk of progression to HGD or EAC in BE patient was firstly determined by the presence or absence of low-grade dysplasia with an odds ratio of 4.2 (2.1-8.5) [47] (Table 2). The risk of progression to HGD or cancer further significantly increased with increasing BE segment length with an odds ratio of 1.2 (1.1-1.3) per additional $\mathrm{cm}$ in length. The study further confirmed that use of a PPI or statin significantly decreased the risk of progression to HGD or cancer, while the protective effect of NSAID use was of borderline significance [47]. Finally, the systematic review confirmed that higher age and male sex are risk factors for disease progression. Although reported as separate factors, various studies have suggested that these are actually interrelated and together a reflection of the duration of presence of BE. Males on average have a lower age of onset of BE, and thus at any given age a higher likelihood of long disease duration. A Dutch cohort study suggested that HGD and cancer in particular occurred after
Table 2 Patient characteristics and risk of neoplastic progression to $\mathrm{HGD} / \mathrm{EAC}$ in Barrett's esophagus

\begin{tabular}{|c|c|c|}
\hline \multicolumn{2}{|l|}{ Patient characteristic } & Risk estimation \\
\hline \multicolumn{2}{|l|}{ Age [47] } & OR 1.03 (95\% CI $1.01 ; 1.05)$ \\
\hline \multicolumn{2}{|l|}{ Male gender [47] } & OR $2.16(95 \%$ CI $1.84 ; 2.53)$ \\
\hline \multicolumn{2}{|c|}{ Length of BE segment (per additional $\mathrm{cm}$ ) [47] } & OR $1.25(95 \%$ CI $1.16 ; 1.36)$ \\
\hline \multicolumn{2}{|c|}{ Proton pump inhibitors [47] } & OR 0.55 (95\% CI $0.32 ; 0.96)$ \\
\hline \multirow[t]{3}{*}{ NSAIDs and statins } & NSAIDs [44] & HR 0.47 (95\% CI 0.24; 0.93) \\
\hline & Statins [47] & OR $0.48(95 \%$ CI $0.31 ; 0.73)$ \\
\hline & Combination [44] & HR 0.22 (95\% CI 0.06; 0.85) \\
\hline \multicolumn{2}{|l|}{ Tobacco smoking [46] } & HR 2.03 (95\% CI 1.29; 3.17) \\
\hline \multicolumn{2}{|c|}{ Low-grade dysplasia [47] } & OR 4.25 (95\% CI $2.58 ; 7.0)$ \\
\hline
\end{tabular}


an "incubation period" of at least 30 years [24]. A British study suggested a 17-year delay in development of BE in women compared to men [48]. Together, these phenomena can explain male gender and age as risk factors for progression to HGD and cancer [47].

\section{Population Picture}

Together, these data allow constructing population overviews. This provides a picture with GERD as most common clinical diagnosis in Western countries. A distinct proportion of them develops Barrett's esophagus. We do not see Barrett's metaplasia gradually develop over time in GERD patients undergoing repeat endoscopy, but we do know that the prevalence of BE steadily increases with age with a time gap between men and women. Data on the prevalence of BE in symptomatic patients undergoing diagnostic endoscopy are by definition biased. However, studies into the prevalence of BE in other populations such as subjects undergoing screening colonoscopy, and true population studies evaluating the prevalence of $\mathrm{BE}$ in unselected subjects provide more reliable information [16-21]. These studies reported a rather steady $3-10 \%$ prevalence of BE, with some variation above and below these borders. The differences without doubt in part relate to the population and age-group studied, as well as to the definition of BE. Some studies for instance reported on the prevalence of endoscopic signs of BE metaplasia and the histopathological prevalence of, respectively, columnar metaplasia and specialized intestinal metaplasia. If we would accept the 7\% prevalence of BE among Dutch 50+ year olds as previously observed in colonoscopy screenees, this would translate to approximately $308,000 \mathrm{BE}$ carriers among 4.4 million subjects. With an approximate 1500 esophageal adenocarcinomas per year in the same population, the annual incidence of EAC among all BE carriers would approximate $0.49 \%$.

Another approach can be based on all identified BE cases in the Netherlands, in combination with the fact that we know that no more than 5-10\% of EAC patients had been known with BE prior to their cancer diagnosis [49]. In the Netherlands in 2010, close to 42,500 subjects had been identified by histology with BE [50]. If these indeed represented a maximal $10 \%$ of all BE cases, their total would amount to at least 425,000 . Assuming that these cases in particular affect subjects aged 50 years and above, it would be equivalent with a $9 \%$ prevalence of $\mathrm{BE}$ in this subgroup of the total population. The annual 1500 cases would then be equivalent to an annual incidence of EAC among all BE carriers of approximately $0.35 \%$.

One quickly realizes that both combinations are rough estimates. The beauty is that they allow providing an overall population picture that then can be used for public health discussions. The picture also makes clear that a $1 \%$ population prevalence of $\mathrm{BE}$ as sometimes suggested does not match cancer incidence data. Again, for the Dutch situation this would match 44,000 BE cases among 4.4 million 50+ year olds. This would imply that nearly all BE cases have been identified in this country, and that the cancer incidence among these BE cases would approximate 3\% per year, or six- to tenfold the rate of any cohort study with the exception of studies on HGD. Both implications are unlikely and not supported by the literature. These epidemiological exercises also illustrate the correlation between population prevalence of BE, cancer incidences among BE cases, and resulting EAC cases per year, with the latter number being the most solid and based on cancer registries. The given number of cancer cases thus translates back in the interrelated BE prevalence and cancer incidence.

These data were in recent years supported by three large population studies (Table 3 ). The first and largest came from the Netherlands and reported on 42,207 BE patients identified in the Dutch national histopathology registry [50]. Among patients undergoing endoscopic surveillance, the annual incidence of EAC was $0.4 \%$. However, the cancer incidence was $0.14 \%$ overall for the total cohort when also including all BE patients who did not undergo surveillance. Two similar studies with, respectively, 11,000 Irish and 8500 Danish patients had very similar results with cancer incidences of 0.13 and $0.15 \%$ [51, 52]. These consistent data from three population studies in Western Europe with large patient numbers suggest that the overall incidence of EAC in unselected $\mathrm{BE}$ cases is low. As mentioned, this matches with a high prevalence of $\mathrm{BE}$ in the general population.

\section{Natural History of BE and Benefits of Screening and Surveillance}

These data are very relevant to determine optimal cancer prevention strategies. Modeling studies for instance showed that the cost efficacy of BE surveillance strongly depended
Table 3 Population-based incidence studies of esophageal adenocarcinoma (EAC) in unselected patients with Barrett's esophagus

\begin{tabular}{lcclll}
\hline Country & BE patients $(n)$ & $\begin{array}{l}\text { Incident } \\
\text { EAC }(n)\end{array}$ & $\begin{array}{l}\text { Person-years } \\
\text { follow-up }\end{array}$ & $\begin{array}{l}\text { EAC incidence } \\
\text { \% per year })\end{array}$ & $\begin{array}{l}95 \% \\
\text { confidence } \\
\text { interval }\end{array}$ \\
\hline The Netherlands [50] & 42,207 & 337 & 234,821 & 0.14 & $0.12-0.16$ \\
Ireland [51] & 8522 & 79 & 59,784 & 0.13 & $0.10-0.16$ \\
Denmark [52] & 11,028 & 66 & 56,782 & 0.12 & $0.09-0.15$ \\
\hline
\end{tabular}


on cancer incidence [53-56]. These modeling studies generally tend to use higher cancer incidences than reported in the above-mentioned population studies. Papers on BE, whether original studies, reviews, or guidelines, often start with statements such as that EAC is the most rapidly increasing cancer in the world [34] and the fastest growing cause of cancer mortality [36]. Statements such as these find a source in the fact that EAC has shown a proportional dramatic increase in Western populations over the past 40 years. However, despite this proportional increase, both statements are untrue and likely to remain untrue for long. When looking at GItract cancers alone, mortality worldwide due to gastric, colorectal, liver, and pancreatic cancer remains far more common than mortality due to esophageal adenocarcinoma. Further, mortality due to colorectal, liver, and pancreatic cancer rose more in absolute numbers in the period of 1990-2013 than mortality due to esophageal cancer [57]. This is true on a global basis, but in Western countries alone. Even in regions such as North America and Western Europe with the highest EAC incidence, it remains a rare malignancy roughly making up for $1 \%$ of all cancers [58]. Unfortunately, 5-year survival rates remain poor with only $20 \%$ [58]. This survival only slowly increased over the past 40 years.

All together, we are thus faced with a major clinical and public health problem. At a time of decreasing prevalence of Helicobacter pylori and increasing prevalence of obesity, we are faced with GERD affecting a substantial part of the adult population worldwide. A considerable proportion of GERD-affected subjects develop BE. This means that many countries will have to take into account that many of their $50+$ year olds carry BE, even when undiagnosed in most of them. A small proportion of these subjects annually progress to HGD and EAC. At an early stage, these conditions can be treated by ablative techniques with limited burden and risks. However, at an advanced stage, EAC requires invasive treatment with considerable burden, costs, and mortality. In recent years, treatment for invasive EAC has shown marked improvements with introduction of presurgical chemoradiotherapy [59]. Further efforts are ongoing to see whether chemoradiotherapy can provide curative options and thus replace surgery in certain EAC patients [60]. Nevertheless, prevention and early detection remain by far the most attractive strategy to manage EAC. Together, the high prevalence of $\mathrm{BE}$ and the low cancer incidence among unselected $\mathrm{BE}$ cases, versus the burden of treatment and low survival of EAC ask for smart strategies to select BE patients who are most likely to benefit from surveillance and intervention. These do not include short-segment BE cases, nor the majority of those without dysplasia [61]. A recent multicenter study based on 2697 BE patients developed a scoring system based on gender, smoking status, BE length, and the presence of low-grade dysplasia to classify patients at low, intermediate, and high risk [62]. During a 7-year follow-up, progression to HGD and EAC occurred in 0.5, 4.6, and $12.3 \%$, respectively.

\section{Conclusions}

With many patients undergoing long-term surveillance and providing consent to use their data to improve our knowledge and benefit others, and many researchers worldwide reporting their analyses of these data, we have obtained a major understanding of the natural history of BE. BE is a common condition, with a prevalence increasing with age in men and women, although with a delay in women. The incidence of HGD and cancer in BE depends on segment length, gender, and age, both likely a marker for the length of presence of $\mathrm{BE}$, the presence or absence of low-grade dysplasia, and other factors such as smoking behavior and use of certain medications such as PPIs, statins, and NSAIDs. Targeted use of surveillance and intervention can impact the incidence and mortality due to EAC and is of major benefit to a subgroup of BE patients. The epidemiology and burden of disease ask for further efforts to develop targeted screening, surveillance, and intervention techniques in coming years.

\section{Key Messages}

- The prevalence of GERD has increased with approximately $50 \%$ in the last 20 years.

- The presence of BE has increased simultaneously, in Western countries leading to an estimated population prevalence reaching 3-10\%.

- GERD, male gender, white race, cigarette smoking, and central adiposity are the most common risk factors for Barrett's esophagus.

- Women develop BE at a later age than men.

- Neoplastic progression in BE depends on age, gender, segment length, use of proton pump inhibitors, NSAIDs and statins, tobacco smoking, and the presence of dysplasia.

\section{Compliance with ethical standards}

Conflict of interest The authors declare that they have no conflicts of interest.

Open Access This article is distributed under the terms of the Creative Commons Attribution-NonCommercial 4.0 International License (http://creativecommons.org/licenses/by-nc/4.0/), which permits any noncommercial use, distribution, and reproduction in any medium, provided you give appropriate credit to the original author(s) and the source, provide a link to the Creative Commons license, and indicate if changes were made. 


\section{References}

1. Peery AF, Dellon ES, Lund J, et al. Burden of gastrointestinal disease in the United States: 2012 update. Gastroenterology. 2012;143:1179.e3-1187.e3.

2. El-Serag HB, Sweet S, Winchester CC, et al. Update on the epidemiology of gastro-oesophageal reflux disease: a systematic review. Gut. 2014;63:871-880.

3. Thrift AP, Kramer JR, Qureshi Z, et al. Age at onset of GERD symptoms predicts risk of Barrett's esophagus. Am J Gastroenterol. 2013;108:915-922.

4. van Blankenstein M, Looman CWN, Johnston BJ, et al. Age and sex distribution of the prevalence of Barrett's esophagus found in a primary referral endoscopy center. Am J Gastroenterol. 2005; 100:568-576.

5. Runge TM, Abrams JA, Shaheen NJ. Epidemiology of Barrett's esophagus and esophageal adenocarcinoma. Gastroenterol Clin North Am. 2015;44:203-231.

6. Verbeek RE, Spittuler LF, Peute A, et al. Familial clustering of Barrett's esophagus and esophageal adenocarcinoma in a European cohort. Clin Gastroenterol Hepatol. 2014;12:1656.e1-1663. e1.

7. Gharahkhani P, Fitzgerald RC, Vaughan TL, et al. Genome-wide association studies in oesophageal adenocarcinoma and Barrett's oesophagus: a large-scale meta-analysis. Lancet Oncol. 2016;17:1363-1373.

8. Palles C, Chegwidden L, Li X, et al. Polymorphisms near TBX5 and GDF7 are associated with increased risk for Barrett's esophagus. Gastroenterology. 2015;148:367-378.

9. Sun X, Elston RC, Barnholtz-Sloan JS, et al. Predicting Barrett's esophagus in families: an esophagus translational research network (BETRNet) model fitting clinical data to a familial paradigm. Cancer Epidemiol Biomarkers Prev. 2016;25:727-735.

10. Dong J, Buas MF, Gharahkhani P, et al. Determining risk of Barrett's esophagus and esophageal adenocarcinoma based on epidemiologic factors and genetic variants. Gastroenterology. 2018;154:1273-1281.

11. Fock KM, Talley N, Goh KL, et al. Asia-Pacific consensus on the management of gastro-oesophageal reflux disease: an update focusing on refractory reflux disease and Barrett's oesophagus. Gut. 2016;65:1402-1415.

12. Cameron AJ, Zinsmeister AR, Ballard DJ, et al. Prevalence of columnar-lined (Barrett's) esophagus. Comparison of population-based clinical and autopsy findings. Gastroenterology. 1990;99:918-922.

13. van Soest EM, Dieleman JP, Siersema PD, et al. Increasing incidence of Barrett's oesophagus in the general population. Gut. 2005;54:1062-1066.

14. Masclee GMC, Coloma PM, de Wilde M, et al. The incidence of Barrett's oesophagus and oesophageal adenocarcinoma in the United Kingdom and the Netherlands is levelling off. Aliment Pharmacol Ther. 2014;39:1321-1330.

15. Shiota S, Singh S, Anshasi A, et al. Prevalence of Barrett's esophagus in Asian countries: a systematic review and meta-analysis. Clin Gastroenterol Hepatol. 2015;13:1907-1918.

16. Rex DK, Cummings OW, Shaw M, et al. Screening for Barrett's esophagus in colonoscopy patients with and without heartburn. Gastroenterology. 2003;125:1670-1677.

17. Ward EM, Wolfsen HC, Achem SR, et al. Barrett's esophagus is common in older men and women undergoing screening colonoscopy regardless of reflux symptoms. Am J Gastroenterol. 2006;101:12-17.

18. Zagari RM, Eusebi LH, Rabitti S, et al. Prevalence of upper gastrointestinal endoscopic findings in the community: a systematic review of studies in unselected samples of subjects. $J$ Gastroenterol Hepatol. 2016;31:1527-1538.

19. Ronkainen J, Aro P, Storskrubb T, et al. Prevalence of Barrett's esophagus in the general population: an endoscopic study. Gastroenterology. 2005;129:1825-1831.

20. Zagari RM, Fuccio L, Wallander M-A, et al. Gastro-oesophageal reflux symptoms, oesophagitis and Barrett's oesophagus in the general population: the Loiano-Monghidoro study. Gut. 2008;57:1354-1359.

21. Zou D, He J, Ma X, et al. Epidemiology of symptom-defined gastroesophageal reflux disease and reflux esophagitis: the systematic investigation of gastrointestinal diseases in China (SILC). Scand J Gastroenterol. 2011;46:133-141.

22. van Soest EM, Siersema PD, Dieleman JP, et al. Age and sex distribution of the incidence of Barrett's esophagus found in a Dutch primary care population. Am J Gastroenterol. 2005;100:2599-2600.

23. Coleman HG, Bhat S, Murray LJ, et al. Increasing incidence of Barrett's oesophagus: a population-based study. Eur J Epidemiol. 2011;26:739-745.

24. den Hoed CM, van Blankenstein M, Dees J, et al. The minimal incubation period from the onset of Barrett's oesophagus to symptomatic adenocarcinoma. Br J Cancer. 2011;105:200-205.

25. Thomas T, Abrams KR, De Caestecker JS, et al. Meta analysis: cancer risk in Barrett's oesophagus. Aliment Pharmacol Ther. 2007;26:1465-1477.

26. Yousef F, Cardwell C, Cantwell MM, et al. The incidence of esophageal cancer and high-grade dysplasia in Barrett's esophagus: a systematic review and meta-analysis. Am J Epidemiol. 2008;168:237-249.

27. Sikkema M, de Jonge PJF, Steyerberg EW, et al. Risk of esophageal adenocarcinoma and mortality in patients with Barrett's esophagus: a systematic review and meta-analysis. Clin Gastroenterol Hepatol. 2010;8:235-244.

28. Qiao Y, Hyder A, Bae SJ, et al. Surveillance in patients with Barrett's esophagus for early detection of esophageal adenocarcinoma: a systematic review and meta-analysis. Clin Transl Gastroenterol. 2015;6:e131.

29. Qumseya BJ, Wani S, Gendy S, et al. Disease progression in Barrett's low-grade dysplasia with radiofrequency ablation compared with surveillance: systematic review and meta-analysis. Am J Gastroenterol. 2017;112:849-865.

30. Desai TK, Krishnan K, Samala N, et al. The incidence of oesophageal adenocarcinoma in non-dysplastic Barrett's oesophagus: a meta-analysis. Gut. 2012;61:970-976.

31. Thota PN, Vennalaganti P, Vennelaganti S, et al. Low risk of highgrade dysplasia or esophageal adenocarcinoma among patients With Barrett's esophagus less than $1 \mathrm{~cm}$ (irregular Z line) within 5 years of index endoscopy. Gastroenterology. 2017;152:987-992.

32. Singh S, Manickam P, Amin AV, et al. Incidence of esophageal adenocarcinoma in Barrett's esophagus with low-grade dysplasia: a systematic review and meta-analysis. Gastrointest Endosc. 2014;79:897.e4-909.e4.

33. Kestens C, Offerhaus GJA, van Baal JWPM, et al. Patients with Barrett's esophagus and persistent low-grade dysplasia have an increased risk for high-grade dysplasia and cancer. Clin Gastroenterol Hepatol. 2016;14:956.e1-962.e1.

34. Phoa KN, van Vilsteren FGI, Weusten BLAM, et al. Radiofrequency ablation vs endoscopic surveillance for patients with Barrett esophagus and low-grade dysplasia: a randomized clinical trial. JAMA. 2014;311:1209-1217.

35. Rastogi A, Puli S, El-Serag HB, et al. Incidence of esophageal adenocarcinoma in patients with Barrett's esophagus and high-grade dysplasia: a meta-analysis. Gastrointest Endosc. 2008;67:394-398. 
36. Bennett C, Vakil N, Bergman J, et al. Consensus statements for management of Barrett's dysplasia and early-stage esophageal adenocarcinoma, Based on a Delphi process. Gastroenterology. 2012;143:336-346.

37. Fitzgerald RC, di Pietro M, Ragunath K, et al. British Society of Gastroenterology guidelines on the diagnosis and management of Barrett's oesophagus. Gut. 2014;63:7-42.

38. Shaheen NJ, Falk GW, Iyer PG, et al. ACG clinical guideline: diagnosis and management of Barrett's esophagus. Am J Gastroenterol. 2016;111:30-50.

39. Anaparthy R, Gaddam S, Kanakadandi V, et al. Association between length of Barrett's esophagus and risk of high-grade dysplasia or adenocarcinoma in patients without dysplasia. Clin Gastroenterol Hepatol. 2013;11:1430-1436.

40. Sikkema M, Looman CWN, Steyerberg EW, et al. Predictors for neoplastic progression in patients with Barrett's esophagus: a prospective cohort study. Am J Gastroenterol. 2011;106:1231-1238.

41. Kastelein F, Spaander MCW, Steyerberg EW, et al. Proton pump inhibitors reduce the risk of neoplastic progression in patients with Barrett's esophagus. Clin Gastroenterol Hepatol. 2013;11:382-388.

42. Singh S, Garg SK, Singh PP, et al. Acid-suppressive medications and risk of oesophageal adenocarcinoma in patients with Barrett's oesophagus: a systematic review and meta-analysis. Gut. 2014;63:1229-1237.

43. Nguyen DM, Richardson P, El-Serag HB. Medications (NSAIDs, statins, proton pump inhibitors) and the risk of esophageal adenocarcinoma in patients with Barrett's esophagus. Gastroenterology. 2010;138:2260-2266.

44. Kastelein F, Spaander MCW, Biermann K, et al. Nonsteroidal anti-inflammatory drugs and statins have chemopreventative effects in patients with Barrett's esophagus. Gastroenterology. 2011;141:2000-2008.

45. Nguyen T, Duan Z, Naik AD, et al. Statin use reduces risk of esophageal adenocarcinoma in US veterans with Barrett's esophagus: a nested case-control study. Gastroenterology. 2015;149:1392-1398.

46. Coleman HG, Bhat S, Johnston BT, et al. Tobacco smoking increases the risk of high-grade dysplasia and cancer among patients with Barrett's esophagus. Gastroenterology. 2012;142:233-240.

47. Krishnamoorthi R, Singh S, Ragunathan K, et al. Factors associated with progression of Barrett's esophagus: a systematic review and meta-analysis. Clin Gastroenterol Hepatol. 2017.

48. Derakhshan MH, Liptrot S, Paul J, et al. Oesophageal and gastric intestinal-type adenocarcinomas show the same male predominance due to a 17 year delayed development in females. Gut. 2009;58:16-23.
49. Bhat SK, McManus DT, Coleman HG, et al. Oesophageal adenocarcinoma and prior diagnosis of Barrett's oesophagus: a population-based study. Gut. 2015;64:20-25.

50. de Jonge PJF, van Blankenstein M, Looman CWN, et al. Risk of malignant progression in patients with Barrett's oesophagus: a Dutch nationwide cohort study. Gut. 2010;59:1030-1036.

51. Bhat S, Coleman HG, Yousef F, et al. Risk of malignant progression in Barrett's esophagus patients: results from a large population-based study. J Natl Cancer Inst. 2011;103:1049-1057.

52. Hvid-Jensen F, Pedersen L, Drewes AM, et al. Incidence of adenocarcinoma among patients with Barrett's esophagus. $N$ Engl $J$ Med. 2011;365:1375-1383.

53. Kastelein F, van Olphen S, Steyerberg EW, et al. Surveillance in patients with long-segment Barrett's oesophagus: a cost-effectiveness analysis. Gut. 2015;64:864-871.

54. Gordon LG, Mayne GC, Hirst NG, et al. Cost-effectiveness of endoscopic surveillance of non-dysplastic Barrett's esophagus. Gastrointest Endosc. 2014;79:242.e6-256.e6.

55. Benaglia T, Sharples LD, Fitzgerald RC, et al. Health benefits and cost effectiveness of endoscopic and nonendoscopic cytosponge screening for Barrett's esophagus. Gastroenterology. 2013;144:62. e6-73.e6.

56. Inadomi JM. Cost considerations in implementing a screening and surveillance strategy for Barrett's oesophagus. Best Pract Res Clin Gastroenterol. 2015;29:51-63.

57. Karagiannis-Voules DA, Biedermann P, Ekpo UF, et al. Global, regional, and national age-sex specific all-cause and cause-specific mortality for 240 causes of death, 1990-2013: a systematic analysis for the global burden of disease study 2013. Lancet. 2014;385:117-171.

58. Jemal A, Ward EM, Johnson CJ, et al. Annual report to the nation on the status of cancer, 1975-2014, featuring survival. J Natl Cancer Inst. 2017. https://doi.org/10.1093/jnci/djx030.

59. van Hagen P, Hulshof MCCM, van Lanschot JJB, et al. Preoperative chemoradiotherapy for esophageal or junctional cancer. $N$ Engl J Med. 2012;366:2074-2084.

60. Noordman BJ, Shapiro J, Spaander MC, et al. Accuracy of detecting residual disease after cross neoadjuvant chemoradiotherapy for esophageal cancer (preSANO Trial): rationale and protocol. JMIR Res Protoc. 2015;4:e79.

61. de Jonge PJF, van Blankenstein M, Grady WM, et al. Barrett's oesophagus: epidemiology, cancer risk and implications for management. Gut. 2014;63:191-202.

62. Parasa S, Vennalaganti S, Gaddam S, et al. Development and validation of a model to determine risk of progression of Barrett's esophagus to neoplasia. Gastroenterology. 2018;154:1282-1289. 